\title{
Relationships Between Perceived Value, Satisfaction, and Loyalty of Tourists in Ancient Villages-Taking the Zili Village in Kaiping City as an Example
}

\author{
Yang Sen ${ }^{1, a,{ }^{*}}$, Zhang Ling ${ }^{1, b}$ \\ ${ }^{1}$ Guangdong University of Finance and Economics, School of Geography and \\ Tourism, Guangzhou China \\ aemail: 1028943403@qq.com, bemail: 1085060051@qq.com \\ ${ }^{*}$ Corresponding author
}

Keywords: Perceived value, Tourists' satisfaction, Loyalty, Ancient village.

\begin{abstract}
It is an important topic for all the tourist destination to cultivate loyal tourists as the competition of them becomes more and more intense. This paper mainly discusses the causality among the perceived value, satisfaction and loyalty of tourists who visit ancient villages. The Zili village of Kaiping in Guangdong province is took as an example to investigate the relationship among them. 265 effective questionnaires were collected and the data was analyzed with structural equation model. The analysis suggests that the perceived value of tourists consists of four dimensions: social value, emotional value, brand value and functional value. The perceived value of different dimensions has different influence on tourists' satisfaction and loyalty. Social value and emotional value have significant positive influence on tourists' satisfaction and loyalty while the functional value only has significant positive influence on their satisfaction, but the emotional value only has significant positive influence on their loyalty. Tourists' satisfaction is positively correlated with their loyalty. The satisfaction of tourists plays a part intermediary role among social value, emotional value, brand value and loyalty, while the functional value and loyalty have a complete intermediary function among them.
\end{abstract}

\section{古村落游客感知价值、满意与忠诚的关系研究 一以开平市自力古村落为例

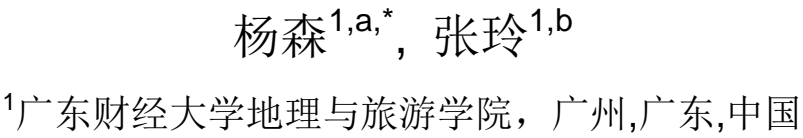 \\ aemail: 1028943403@qq.com, bemail: 1085060051@qq.com \\ “通讯作者}

关键词: 感知价值; 旅游者满意; 忠诚; 古村落

中文摘要. 伴随旅游目的地竞争日益激烈, 如何培育忠诚游客成为旅游目的地面临的重大课 题。论文主要探讨了古村落游客感知价值、满意与忠诚三者之间的因果关系。论文以广东省 开平市自力村为例, 对古村落游客进行了问卷调查, 收集了 265 份有效问卷, 并运用结构方程 模型分析数据。研究发现: 游客感知价值由四个维度组成, 分别为社会价值、情感价值、品 牌价值和功能价值; 不同维度感知价值对游客满意和忠诚影响不同，社会价值和情感价值均 对游客满意和忠诚有显著的正向影响，而功能价值仅对游客满意有显著的正向影响，情感价 
值仅对忠诚有显著正向影响; 游客满意与忠诚呈正相关; 游客满意在社会价值、情感价值和 品牌价值和忠诚之间起着部分中介的作用, 而在功能价值和忠诚的之间起着完全中介的作用。

\section{1. 引言}

十九大报告提出乡村振兴战略, 要让我国优秀传统文化在传统村落得到传承弘扬, 让传 统村落的产业经济得到繁荣发展并为乡村振兴提供动力, 让传统村落的广大农民群众特别是 贫困人口得到长期稳定就业。发展乡村旅游成为实现乡村振兴的一个重要方面。近年来, 越 来越多城市居民到乡村缓解压力、放松身心、暂时远离繁忙的工作和享受殹意的慢生活, 乡 村旅游成为比较受欢迎的旅游类型之一。古村落旅游作为乡村旅游体系中一个比较独特的类 型, 以其深厚的文化底蕴、淳朴的民风和古香古色的建筑遗迹等受到游客的喜爱。但随着我 国古村落旅游的快速发展，一些古村落出现了原真性文化遗失、商业气息浓厚、古建筑的毁 坏与重建现代化的建筑, 使得古村落游客旅游感知价值下降、满意度降低。为了促进我国古 村落旅游及乡村旅游的发展, 如何提高我国古村落旅游的游客感知价值和满意, 进而提升游 客忠诚成为我国现阶段古村落旅游发展中亟待解决的问题。

现有研究表明, 多数研究认同游客感知价值是多维的, 但维度划分众说不一。影响游客 忠诚的关键因素还存在一定的分歧，游客感知价值与满意之间因果关系尚未达成一致。仅有 少数学者关注古村落游客满意与忠诚的关系研究。因此, 本文试图以广东省古村落自力村为 例, 研究游客感知价值、满意与忠诚之间的相互关系。

自力村位于广东开平市塘口镇，是国家重点文物保护单位，广东省唯一的世界文化遗产。 自力村保留了 15 座造型精美、风格各异、中西合璧的碉楼, 是广东乃至全国华侨建筑文化的 典型代表。该村落碉楼主要建于二十世纪初期, 主要用于防匪、防涝及居住, 将中国传统乡 村建筑文化与西洋文化完美的融合在一起，是中西文化交流出现在中国乡村的杰出代表。

\section{2. 研究综述}

\section{1 游客感知价值的研究}

现有关于游客感知价值的研究主要集中在游客感知价值的含义和维度构成上。Zeithaml 认为, 顾客感知价值是基于利得与利失的感知对产品效用所做的总体评价 ${ }^{[1]}$; Sheth等提出如 果把顾客感知价值仅仅认为是质量和价格之间的权衡就过于简单化了,其实任何产品或服务 所提供的价值都包括功能性价值、社会性价值、情感性价值、认知价值和情景价值 ${ }^{[2]}$; Woodruff 提出“顾客价值是顾客对特定使用情景下有助于（有碍于）实现自己目标和目的的产品属性、 这些属性的实效以及使用的结果所感知的偏好与评价”[3]。

多数研究将顾客感知价值的概念视为多维结构 ${ }^{[4]}$ 。由于感知价值是一种主观和动态的结 构, 在不同的顾客、文化和时间中不同, 因此有必要包括消费者头脑中产生的主观或情感反 应 ${ }^{[4]}$ 。Sweeney等人确定了感知价值的五个维度, 即社会价值（可接受性）、情感价值、功能 价值（价格/货币价值）、功能价值（绩效/质量）和功能价值（多功能性） ${ }^{[5]}$; Petrick开发感

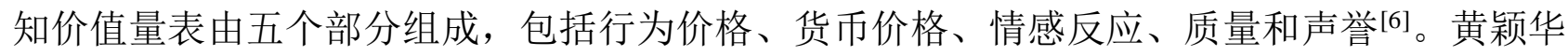
和黄福才构建旅游者感知价值的结构模型并进行验证, 研究发现, 旅游者感知价值包括感知 质量、情感价值、社会价值、感知经济成本、感知非货币成本等维度, 其中情感价值维度的 重要性超过了感知旅游质量 ${ }^{[7]}$ 。王莉等提出了一个由环境价值、特色价值、服务价值、管理 价值、知识教育价值和成本价值组成的游客感知价值 ${ }^{[8]}$ 。Prebensen等研究显示, 游客感知价 值应包括实体价值、社会价值、情感价值、学习价值、经济价值 ${ }^{[9]}$ 。个别学者对顾客感知价 值的驱动因素进行研究, Parasuraman提出顾客价值的驱动因素主要由产品质量、服务质量和 价格因素构成 ${ }^{[10]}$; 白长虹提出产品属性、服务质量、价格、品牌、顾客关系等是顾客价值的 来源要素 ${ }^{111]}$ 。尽管这些研究提供了感知价值的认知和情感维度存在的实证证据, 但对于感知 
价值量表或维度没有一个公认的结构。本论文采用多数学者比较认同的感知价值的五个维度, 即社会价值、情感价值、品牌价值、功能价值、货币价值。

\section{2 游客感知价值与满意关系研究}

顾客满意多数研究采用期望不一致理论，以游客所体验到或感受到的产品或服务与其期 望进行比较: 如果接受到的产品或服务高于其预期, 游客会非常满意; 如果大致相等会满意; 如果低于其期望会不满意。无论是初游者还是再访者都会对旅游目的地有一定期望, 在目的 地感知体验过后才会做出满意与否的情感判断。

多数学者支持价值满意因果链, 即游客感知价值是满意的重要前因。换言之, 满意能很 好地预测未来的行为倾向, 而顾客价值则是满意的主要前因 ${ }^{[12]}$ 。个别研究认为, 与顾客满意 相比较, 感知价值属于较高层次的概念, 具有较为稳定的逻辑判断, 是顾客购后评价行为的 结果 ${ }^{[13]}$ 。

\section{因此，论文提出以下假设:}

$\mathrm{H} 1$ ：游客感知价值正向影响游客满意。

H1-a:社会价值对游客满意有显著的正向影响。

H1-b:情感价值对游客满意有显著的正向影响。

H1-c: 品牌价值对游客满意有显著的正向影响。

H1-d:功能价值对游客满意有显著的正向影响。

H1-e:货币价值对游客满意有显著的正向影响。

\section{3 游客感知价值与忠诚关系研究}

目前对于游客忠诚的概念内涵研究存在分歧。Day提出忠诚是一个包含“行为忠诚 (behavioral loyalty)”和“态度忠诚(attitudinal loyalty)”的“二维结构(two dimensional construct)”, 即“品牌忠诚不仅仅是对同一品牌的连续购买,还应包括态度上的偏好”[14]。Diek和Basu认为，

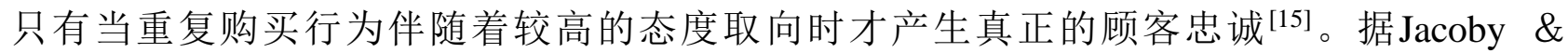
Chestnut的分类法, 品牌忠诚的操作性定义大体上可分为 3 种:行为测量方法、态度测量方法和 综合测量方法(行为和态度的结合 $)^{[16]}$ 。由于重游意向对重游行为具有较好的预测性, 学者们 通常以重游意向代替重游行为 ${ }^{[17]}$ 。本研究采用顾客忠诚的综合测量方法, 从行为忠诚（重游） 与态度忠诚(积极口碑) 两个方面评价游客忠诚。

在感知价值与忠诚关系方面, 学术界还未形成相对一致的看法。部分学者认为感知价值 对忠诚没有直接相关性, Murphy等 ${ }^{[18]}$ 、粟路军和黄福才 ${ }^{[19]}$ 研究发现, 旅游者感知价值对重游 意向没有显著影响。而黄福才和黄颖华研究发现, 感知价值是驱动目的地忠诚最关键的因素 [20], 汪侠等实证得出, 顾客感知质量、顾客感知价值、旅游动机、顾客满意是旅游地顾客忠 诚的 4 个驱动因素,对顾客忠诚均有显著的正向影响 ${ }^{[21]}$ 。郭安禧等认为, 实体价值、经济价值、 学习价值均能显著影响重游意向的形成 ${ }^{[22]}$, Ranjbarian和Pool研究发现, 旅游者获得的感知价 值越高, 今后重返目的地旅游的可能性越大 ${ }^{[23]}$ 。总体而言, 多数研究认同, 旅游者感知价值 积极影响旅游者的重游意向 ${ }^{[23-25]}$ 。

因此, 论文提出以下假设:

$\mathrm{H} 2$ : 游客感知价值正向影响游客忠诚。

H2-a:社会价值对游客忠诚有显著的正向影响。

H2-b: 情感价值对游客忠诚有显著的正向影响。

$\mathrm{H} 2-\mathrm{c}$ : 品牌价值对游客忠诚有显著的正向影响。

H2-d:功能价值对游客忠诚有显著的正向影响。

H2-e:货币价值对游客忠诚有显著的正向影响。 


\section{4 游客满意和忠诚的关系研究}

游客满意是旅游产品或服务的感知绩效与旅游者的心理期望比较的结果，如果前者高于 后者, 旅游者就会感到满意; 相反, 就会感到不满意 ${ }^{[26]}$ 。粟路军和黄福才研究发现, 无论是

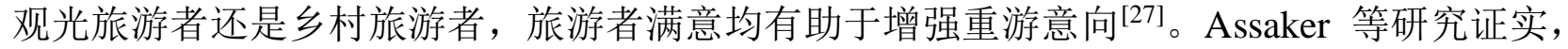
旅游者满意对短期、中期、长期重游意向均有显著正向影响 ${ }^{[28]}$ 。Chi和Qu的研究证实了目的 地属性满意度和总体满意度是目的地忠诚的直接驱动变量 ${ }^{[29]}$ 。但Mechinda等人对到访泰国清 迈(Chiangmai)的非商务型重游者的调查发现, 满意度对游客行为忠诚的影响作用非常有限 [30]。韩经纶等研究发现服务质量决定了顾客满意, 但顾客满意却不一定必然导致顾客忠诚 ${ }^{[31]}$ 。 因此, 论文提出以下假设:

$\mathrm{H} 3$ ：游客满意对忠诚有显著的积极影响。

\section{5 游客感知价值、满意与忠诚的关系研究}

部分学者认为满意在游客感知价值对忠诚的关系中起中介作用, 但也有部分学者发现满 意的中介作用不显著。根据“认知-情感-意向”关系理论, 认知、情感、意向之间是相互关联的, 同时存在层级关系, 情感在认知与意向之间起着中介作用 ${ }^{[32]}$ 。根据该理论, 旅游者感知价值 属于“认知”范畴, 旅游者满意属于“情感”范畴, 重游意向属于“意向”范畴。有研究表明, 游 客感知价值对满意有显著正向影响 ${ }^{[33]}$, 而满意又对重游意向有显著正向影响 ${ }^{[34]}$ 。Quintal和 Polczynski ${ }^{[35]}$ 、Kim 等 ${ }^{[24]}$ 、Ranjbarian 和 $\mathrm{Pool}^{[23]}$ 等的实证研究均表明, 旅游者感知价值会通 过旅游者满意的中介传导对重游意向产生影响。李文兵发现, 古村落游客感知价值作为单维 度结构通过满意间接正面影响忠诚的形成,满意则作为中间变量直接正面影响忠诚的形成 ${ }^{[36]}$; Um等 ${ }^{[37]}$ 人、Hutchinson等 ${ }^{[38]}$ 人和Gallzrza \& Saura ${ }^{[39]}$ 证实了感知价值通过满意度的中介作用 对目的地忠诚所具有的间接影响效果; 高水平的感知价值会使顾客对旅游景区非常满意, 进 而产生顾客忠诚 ${ }^{[40]}$; 部分学者发现感知价值正向影响满意, 但是对游客忠诚正向影响不显著, 满意正向影响游客忠诚 ${ }^{[41]}$ 。

因此，论文提出以下假设:

$\mathrm{H} 4$ ：游客满意在感知价值与游客忠诚之间具有中介作用。

H4-a:游客满意在社会价值与游客忠诚之间具有中介作用。

$\mathrm{H} 4-\mathrm{b}:$ 游客满意在情感价值与游客忠诚之间具有中介作用。

H4-c:游客满意在品牌价值与游客忠诚之间具有中介作用。

H4-d:游客满意在功能价值与游客忠诚之间具有中介作用。

H4-e:游客满意在货币价值与游客忠诚之间具有中介作用。

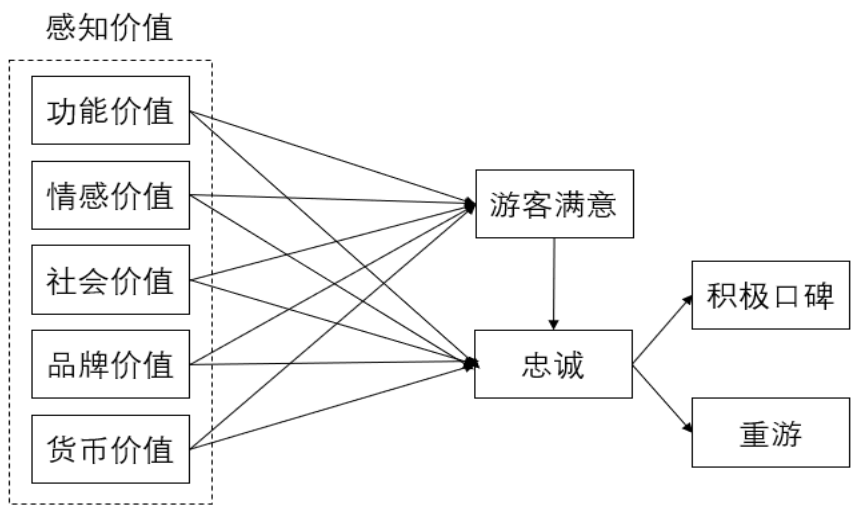

图1 本研究的概念模型

根据以上研究假设, 本研究得出感知价值、满意与忠诚之间因果关系的概念模型图, 具 体如图1。其中自变量为感知价值（功能价值、情感价值、社会价值品牌价值和货币价值）, 中介变量为游客满意, 因变量为游客忠诚。 


\section{3. 研究方法}

本研究采用自结构问卷法收集数据。调查问卷由 3 个部分组成, 第一部分主要是古村落游 客感知价值和忠诚的调查, 感知价值问题在前人研究的基础上, 从功能价值、货币价值、情 感价值、社会价值、品牌价值 5 个方面 29 个题项进行测量, 行为意图包括 2 个题项。第二部分 是游客满意的调查, 共有 2 个题项。第一、二部分均采用李克特五级量表进行测量, 从 1 到 5 分别表示非常不同意、不同意、一般、同意、非常同意, 或者非常不满意、不满意、一般、 满意和非常满意。第三部分是样本的人口学特征调查, 包括性别, 年龄, 居住地, 职业, 受 教育程度, 来游次数, 目的地信息来源和来访方式, 具体见表 1 。

表1 量表的结构、题项和来源

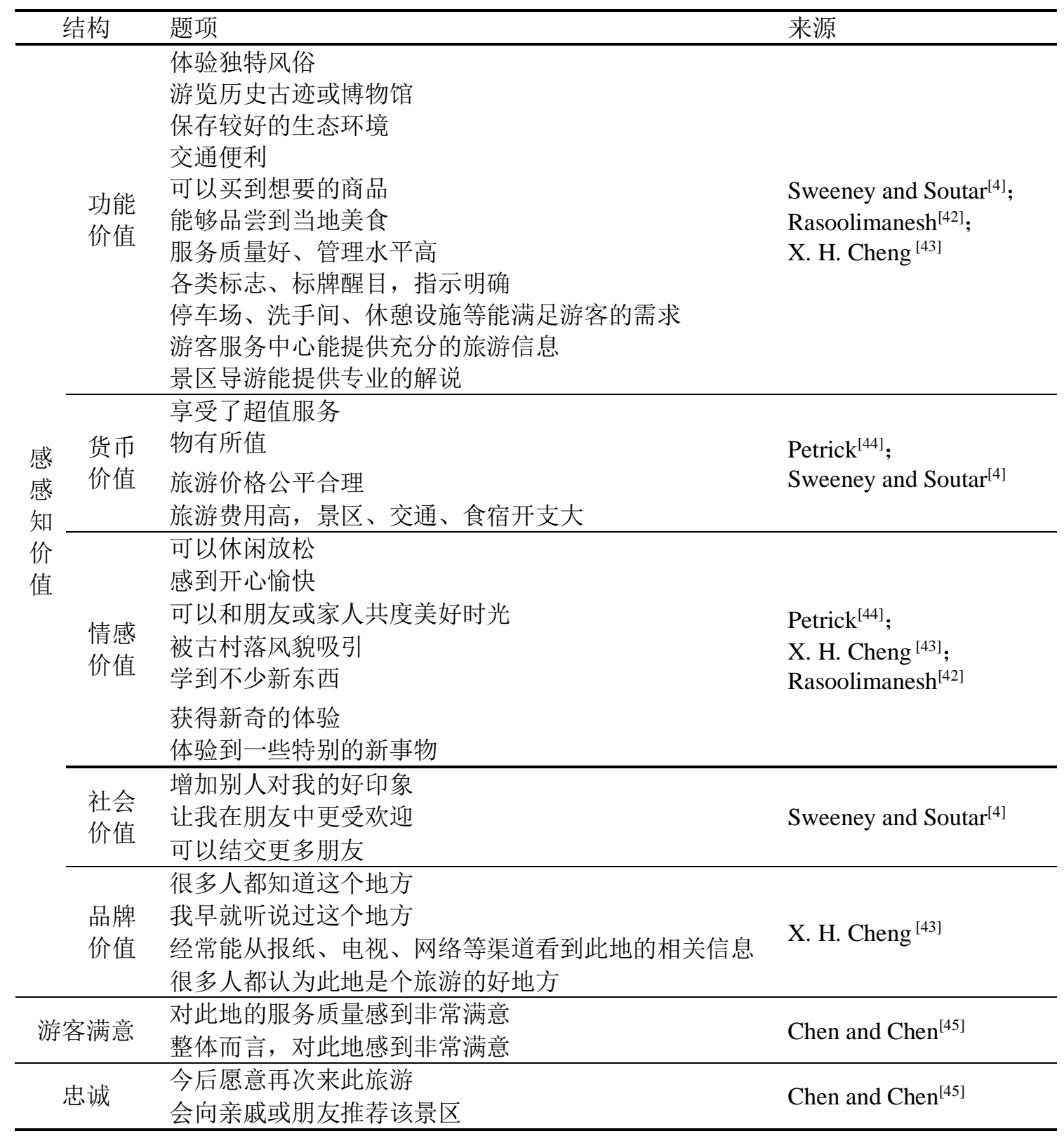

调查地点为广东省开平市自力村。调查分为两次完成，第一次调查时间为 2017年6月 24-25日, 第二次调查时间为 2018年5月1-3日; 问卷采取随机抽样方式发放, 由游客自己填 答然后访问者回收，两次共计发放问卷 300 份，有效回收问卷 265 份，有效回收率为 $88.33 \%$ 。 本研究运用SPSS25.0和Amos24.0软件进行了数据分析。 


\section{4. 研究结果}

\section{1 人口统计分析}

在所有被调查的游客中, 男性略多于女性, 其中男性占 $54.3 \%$ 。年龄在 25 岁以下 $(30.6 \%)$ 和36-45岁 (29.1\%) 占有相对优势。游客主要来源于广州周边城市, 占被调查的游客总数的 $38.1 \%$, 其次是广东省其他城市占 $29.1 \%$; 从职业来看, 以企业人员 $(39.2 \%)$ 和学生 $(23.4 \%)$ 为主。受教育程度以大专或本科比重最大, 为 $64.5 \%$ 。在所有被调查者当中, 初游者占到 $84.2 \%$ 。 从目的地信息来源来看, 以网络媒体最高, 占所有被调查者总数的 $35.8 \%$, 其次是亲朋好友 介绍的占 $34.0 \%$ 。 $71.7 \%$ 游客与家人或朋友一起来的, 具体见表 2 。

表2 样本人口统计学特征

\begin{tabular}{|c|c|c|c|c|c|}
\hline 变量 & 类别 & 比例 $(0 \%)$ & 奕昌 & 类别 & $\begin{array}{l}\mathrm{N}=265 \\
\text { 例 }(0<)\end{array}$ \\
\hline \multirow[b]{2}{*}{ 性别 } & 男 & 54.3 & \multirow{3}{*}{$\begin{array}{c}\text { 受教育 } \\
\text { 程度 }\end{array}$} & 高中或以下 & 28.7 \\
\hline & 女 & 45.7 & & 大专或本科 & 64.5 \\
\hline \multirow{6}{*}{ 年龄 } & 25岁以下 & 30.6 & & 硕士及以上学历 & 6.8 \\
\hline & 25-35岁 & 28.3 & \multirow{3}{*}{$\begin{array}{c}\text { 来游次 } \\
\text { 数 }\end{array}$} & 1次 & 84.2 \\
\hline & 36-45岁 & 29.1 & & 2-3次 & 14.7 \\
\hline & 46-55岁 & 10.9 & & 3次以上 & 1.1 \\
\hline & 56-65岁 & 1.1 & \multirow{4}{*}{$\begin{array}{c}\text { 目的地 } \\
\text { 信息来 } \\
\text { 源 }\end{array}$} & 书刊杂志 & 11.3 \\
\hline & 65岁以上 & 0 & & 网络媒体 & 35.8 \\
\hline \multirow{4}{*}{ 客源地 } & 广州市 & 23.8 & & 亲朋好友介绍 & 34.0 \\
\hline & 广州周边城市 & 38.1 & & 其他 & 18.9 \\
\hline & 广东省其他城市 & 29.1 & \multirow{8}{*}{$\begin{array}{c}\text { 来访方 } \\
\text { 式 }\end{array}$} & 独立前往 & 6.0 \\
\hline & 广东省外 & 9.1 & & 与家人或朋友一起 & 71.7 \\
\hline \multirow{6}{*}{ 职业 } & 公务人员 & 8.7 & & 跟团 & 10.2 \\
\hline & 企业人员 & 39.2 & & 单位组织 & 4.2 \\
\hline & 教师或科研人员 & 7.5 & & 其他 & 7.9 \\
\hline & 自由职业者 & 9.4 & & & \\
\hline & 学生 & 23.4 & & & \\
\hline & 其他 & 11.7 & & & \\
\hline
\end{tabular}

\section{2 探索性因子分析}

论文采用因子分析中的主成分分析法 (Principal Components)，利用正交旋转中的方差 最大法 (VARIMAX) 寻找游客对古村落感知的重要因素。数据分析结果发现, 相互关系矩 阵的巴特利球形检验 (Bartlett Test of Sphericity)显著性水平为0.000, 统计量为3949.874, KMO

（Kaiser-Meyer-Olkin）值为 0.920 , 表明原始变量之间存在相关性适合作因子分析。

论文采用最大方差正交旋转进行因子旋转, 特征值大于 1 被抽取为公因子。主成份分析法 共提取四个公因子, 解释了原始变量 $65.410 \%$ 的总方差。累计方差贡献率大于 $50 \%$, 被认为是 一个比较满意的结果, 四个因子包括 23 个项目总方差的大部分信息。四个公因子依次被命名 为: 情感价值 $\mathrm{EV}$, 社会价值 $\mathrm{SV}$, 功能价值 $\mathrm{FV}$ 和品牌价值 $\mathrm{BV}$ 。因此, 探索性因子分析结果将 感知价值假设中的五个维度修改为四个维度, 并且四个维度Cronbach's $\alpha$ 值在0.816-0.905之间, 大于 0.7 , 说明每个构面的内部一致性都较高, 具体见表 3 。

\section{3 验证性因子分析}

验证性因子分析用来检验感知价值与其社会价值、情感价值、功能价值与品牌价值四个 子构面之间的关系, 以及感知价值各构面的信度和效度情况。模型拟合结果表明, 卡方值 Chi-square $=470.560$, 自由度 $\mathrm{df}=217, \mathrm{P}=.000$ 。卡方自由度比值 $\mathrm{CMIN} / \mathrm{DF}=2.183, \mathrm{GFI}=0.867$, $\mathrm{NFI}=0.884, \mathrm{CFI}=0.933$, RMR $=0.044, \mathrm{RMSEA}=0.067$ 。除了 GFI和NFI以外, 其余指标均满足 推荐值, 表明模型拟合度较好。 
古村落游客感知价值与其四个子构面的验证性因子分析结果表明，四个子构面的组合信 度CR值在0.8106-0.9074之间, 远大于0.6的参考值, 因此可以认为测量量表具有较高的信度。 AVE值介于 0.4966-0.6154之间, 均等于或大于 0.50 的参考值, 说明模型收玫效度较好。平均方 差提取（AVE）均大于 0.5 。这些指标表明，本研究使用的量表具有良好的结构信度和效度， 具体见表4。

\section{表3 三个古村落感知价值探索性因子分析结果}

\begin{tabular}{|c|c|c|c|c|}
\hline 因子/题项 & 因子载荷 & 特征值 & $\begin{array}{l}\text { 累计方差 } \\
\text { 贡献率 }\end{array}$ & $\begin{array}{c}\mathrm{N}=265 \\
\text { 克朗巴 } \\
\text { 哈系数 }\end{array}$ \\
\hline 情感价值EV & & 10.375 & 45.108 & 0.905 \\
\hline 游览历史古迹或博物馆 & 0.555 & & & \\
\hline 保存较好的生态环境 & 0.707 & & & \\
\hline 物有所值 & 0.607 & & & \\
\hline 旅游价格公平合理 & 0.574 & & & \\
\hline 可以休闲放松 & 0.675 & & & \\
\hline 感到开心愉快 & 0.766 & & & \\
\hline 可以和朋友或家人共度美好时光 & 0.741 & & & \\
\hline 被古村落风貌吸引 & 0.717 & & & \\
\hline 社会价值SV & & 1.879 & 53.277 & 0.904 \\
\hline 学到不少新东西 & 0.665 & & & \\
\hline 获得新奇的体验 & 0.657 & & & \\
\hline 体验到一些特别的新事物 & 0.686 & & & \\
\hline 增加别人对我的好印象 & 0.711 & & & \\
\hline 让我在朋友中更受欢迎 & 0.714 & & & \\
\hline 可以结交更多朋友 & 0.810 & & & \\
\hline 功能价值FV & & 1.420 & 59.450 & 0.824 \\
\hline 能够品尝到当地美食 & 0.573 & & & \\
\hline 服务质量好、管理水平高 & 0.733 & & & \\
\hline 各类标志、标牌醒目, 指示明确 & 0.800 & & & \\
\hline 停车场、洗手间、休憩设施等能满足游客需求 & 0.605 & & & \\
\hline 游客服务中心能提供充分的旅游信息 & 0.703 & & & \\
\hline 品牌价值BV & & 1.371 & 65.410 & 0.816 \\
\hline 很多人都知道这个地方 & 0.851 & & & \\
\hline 我早就听说过这个地方 & 0.891 & & & \\
\hline $\begin{array}{l}\text { 经常能从报纸、杂志、电视、网络等渠道看到此 } \\
\text { 地的相关信息 }\end{array}$ & 0.652 & & & \\
\hline 很多人都认为此地是个旅游的好地方 & 0.520 & & & \\
\hline
\end{tabular}

注意: 享受了超值服务; 体验独特风俗; 可以买到想要的商品; 交通便利; 景区导游能提供专业的解说; 旅游 费用高，景区、交通、食宿开支大，由于载荷小于 0.5 , 被删掉。

\section{4 结构方程分析}

论文采用极大似然法（Maximum Likelihood Estimates）对感知价值的四个构面、旅游者 满意和忠诚之间因果关系进行了检验, 概念模型与数据匹配较好 (Chi-square $=610.278, \mathrm{df}=302$, $\mathrm{CMIN} / \mathrm{DF}=2.021, \mathrm{GFI}=0.858, \mathrm{NFI}=0.889, \mathrm{CFI}=0.940, \mathrm{RMSEA}=0.062, \mathrm{RMR}=0.041$ ）。结果 表明: 社会价值、情感价值和功能价值对旅游者满意具有显著的积极影响, 品牌价值对游客 满意没有积极影响, 即H1-a、H1-b和H1-d成立, H1-c不成立; 其中社会价值和功能价值在0.001 水平下显著的，而情感价值是在 0.05 水平下显著的。社会价值、情感价值和品牌价值对游客 忠诚的路径系数分别为 $0.253,0.225$, 和 0.270 , $\mathrm{t}$ 值分别为 $3.016,2.880$ 和 4.926 , 支持假设 $\mathrm{H} 2-\mathrm{a}$, H2-b和H2-c, 但是H2-d被拒绝, 证明了社会价值、情感价值和品牌价值对游客忠诚有显著地 
积极影响, 而功能价值对游客忠诚则有显著的消极影响。旅游者满意对忠诚的路径系数为 $0.468, \mathrm{t}$ 值为 $6.159, \mathrm{H} 3$ 成立，支持旅游者满意对忠诚有显著地积极影响，具体见表5。

论文参照Preacher 和Hayes ${ }^{[46]}$ 和Hayes ${ }^{[47]}$ 提出的Bootstrap 方法对旅游者满意在感知价值四 个维度与游客忠诚之间的中介作用进行检验, 样本量是选择 5000 , 在 $95 \%$ 置信区间下, 中介 检查结果四个维度都不包含 0 （其中情感价值 LLCI $=0.3470, \quad$ ULCI $=0.6930$; 社会价值 $\mathrm{LLCI}=0.3016$, ULCI=0.5869; 功能价值LLCI=0.6100, ULCI $=0.8832$; 品牌价值LLCI=0.2909, $\mathrm{ULCI}=0.5876$ ），表明了游客满意的中介作用显著，且情感价值、社会价值、功能价值和品牌 价值中介效应大小依次为 $0.5117 、 0.4361 、 0.7421$ 和 0.4269 。此外, 控制了中介变量旅游者满 意之后, 自变量感知价值三个维度对因变量游客忠诚的影响显著，区间不包含0（其中情感价 值LLCI $=0.4586, \mathrm{ULCI}=0.7467$; 社会价值LLCI $=0.4113, \mathrm{ULCI}=0.6678$; 品牌价值LLCI $=0.3134$, $\mathrm{ULCI}=0.5396$ ），表明两类效应结果都很显著，可以认为假设H4-a、H4-b、H4-c成立。但是， 功能价值对游客忠诚影响区间内包括 0 , 即LLCI $=-0.0480$, ULCI $=0.2653$, 即功能价值通过旅 游者满意对游客忠诚的直接作用不显著,具体见表 6 。

综上所述, 游客感知价值中的三个维度情感价值、社会价值、品牌价值对游客忠诚的直 接效果显著, 感知价值这一变量通过旅游者满意的中介作用对游客忠诚的间接效应也很显著, 旅游者满意起着部分中介的作用。但是功能价值对游客忠诚间接效应显著, 直接效应不显著, 旅游者满意具有完全中介作用。

表4 感知价值验证性因子分析结果

$\mathrm{N}=265$

\begin{tabular}{|c|c|c|c|c|c|}
\hline 观察变量 & S.E. & $\mathrm{t}$ 值 & $\begin{array}{l}\text { 标准化回归系 } \\
\text { 数 }\end{array}$ & CR & AVE \\
\hline 情感价值EV & & & & 0.9074 & 0.5537 \\
\hline 旅游价格公平合理 & 0.245 & 11.987 & 0.672 & & \\
\hline 物有所值 & 0.216 & 13.851 & 0.747 & & \\
\hline 保存较好的生态环境 & 0.215 & 10.050 & 0.582 & & \\
\hline 游览历史古迹或博物馆 & 0.231 & 11.873 & 0.666 & & \\
\hline 可以休闲放松 & 0.195 & 14.739 & 0.780 & & \\
\hline 感到开心愉快 & 0.186 & 15.884 & 0.821 & & \\
\hline 可以和朋友或家人共度美好时光 & 0.200 & 15.635 & 0.810 & & \\
\hline 被古村落风貌吸引 & 0.210 & 16.449 & 0.836 & & \\
\hline 社会价值SV & & & & 0.9055 & 0.6154 \\
\hline 增加别人对我的好印象 & 0.222 & 15.389 & 0.805 & & \\
\hline 体验到一些特别的新事物 & 0.197 & 16.500 & 0.841 & & \\
\hline 获得新奇的体验 & 0.219 & 15.325 & 0.803 & & \\
\hline 学到不少新东西 & 0.243 & 14.285 & 0.764 & & \\
\hline 让我在朋友中更受欢迎 & 0.241 & 13.963 & 0.755 & & \\
\hline 可以结交更多朋友 & 0.245 & 13.440 & 0.734 & & \\
\hline 功能价值FV & & & & 0.8302 & 0.4966 \\
\hline 各类标志、标牌醒目, 指示明确 & 0.201 & 14.086 & 0.776 & & \\
\hline 服务质量好、管理水平高 & 0.197 & 16.500 & 0.702 & & \\
\hline 能够品尝到当地美食 & 0.228 & 11.003 & 0.646 & & \\
\hline $\begin{array}{l}\text { 停车场、洗手间、休脜设施等能满足游 } \\
\text { 客的需求 }\end{array}$ & 0.247 & 10.258 & 0.611 & & \\
\hline 游客服务中心能提供充分的旅游信息 & 0.194 & 13.985 & 0.773 & & \\
\hline 品牌价值BV & & & & 0.8106 & 0.518 \\
\hline 很多人都认为此地是个旅游的好地方 & 0.229 & 12.382 & 0.749 & & \\
\hline $\begin{array}{l}\text { 经常能从报纸、电视、网络等渠道看到 } \\
\text { 此地的相关信息 }\end{array}$ & 0.221 & 13.435 & 0.767 & & \\
\hline 我早就听说过这个地方 & 0.261 & 11.075 & 0.709 & & \\
\hline 很多人都知道这个地方 & 0.252 & 9.925 & 0.648 & & \\
\hline
\end{tabular}




\section{5. 结论与讨论}

\section{1 研究结论}

本研究构建了古村落游客感知价值对游客忠诚的影响机理模型, 验证了游客感知价值的 多维结构以及如何对游客忠诚产生影响。研究发现:

第一, 验证了古村落旅游游客感知价值的多维结构, 游客感知价值共有四个维度组成, 依次为社会价值、情感价值、品牌价值和功能价值。在广泛参考了以往顾客价值、古村落旅 游以及相关的顾客价值测量量表等相关文献的基础上, 结合古村落旅游行业的背景, 开发设 计了古村落游客感知价值量表, 初始设计游客感知价值为五个维度, 最后研究结果发现古村 落游客感知价值是一个四维的结构。感知价值其中的三个维度, 包括社会价值、情感价值和 功能价值与Sweeney研究的结论基本上一致的 ${ }^{[6]}$ 。另外品牌价值与白长虹的品牌关系比较相似 的 ${ }^{[11]}$ 。由此可见, 游客感知价值是一个多维结构。

表5 结构方程分析结果

\begin{tabular}{ccccc}
\hline 路径 & 标准化回归系数 & T值 & $\mathrm{P}$ & 假设 \\
\hline $\mathrm{SV} \rightarrow \mathrm{SAT}$ & 0.424 & 4.613 & $* * *$ & 支持 \\
$\mathrm{EV} \rightarrow \mathrm{SAT}$ & 0.186 & 2.050 & 0.040 & 支持 \\
$\mathrm{FV} \rightarrow \mathrm{SAT}$ & 0.277 & 3.692 & $* * *$ & 支持 \\
$\mathrm{BV} \rightarrow \mathrm{SAT}$ & 0.058 & 0.911 & 0.362 & 拒绝 \\
$\mathrm{SV} \rightarrow \mathrm{LOY}$ & 0.253 & 3.016 & 0.003 & 支持 \\
$\mathrm{EV} \rightarrow \mathrm{LOY}$ & 0.225 & 2.880 & 0.004 & 支持 \\
$\mathrm{BV} \rightarrow \mathrm{LOY}$ & 0.270 & 4.926 & $* * *$ & 支持 \\
$\mathrm{FV} \rightarrow \mathrm{LOY}$ & -0.197 & -2.927 & 0.003 & 拒绝 \\
$\mathrm{SAT} \rightarrow \mathrm{LOY}$ & 0.468 & 6.159 & $* * *$ & 支持
\end{tabular}

注意: SV为社会价值, $\mathrm{EV}$ 为情感价值, $\mathrm{BV}$ 为品牌价值, $\mathrm{FV}$ 为功能价值, $\mathrm{SAT}$ 为旅游者满意, $\mathrm{LOY}$ 为游客忠诚。 $* * *$ 为 $\mathrm{P}$ 小 0.001 .

表6 中介作用检验结果

\begin{tabular}{|c|c|c|c|c|c|c|}
\hline \multirow{3}{*}{ 路径 } & \multirow{3}{*}{$\begin{array}{c}\text { 点估计 } \\
\text { 值 }\end{array}$} & \multirow{2}{*}{\multicolumn{2}{|c|}{$\begin{array}{l}\text { 系数乘积 } \\
\text { uct of coefficients }\end{array}$}} & \multirow{2}{*}{\multicolumn{2}{|c|}{$\begin{array}{l}\text { Bootstapping (5000) } \\
\text { Bias-corrected 95\% CI }\end{array}$}} & \multirow{3}{*}{$\begin{array}{l}\text { 双尾 } \\
\text { 检验 }\end{array}$} \\
\hline & & & & & & \\
\hline & & $\begin{array}{l}\text { 标准 } \\
\text { 误差 }\end{array}$ & $\mathrm{t}$ 值 & $\begin{array}{c}\text { 下限 } \\
\text { lower }\end{array}$ & $\begin{array}{l}\text { 上限 } \\
\text { upper }\end{array}$ & \\
\hline \multirow[t]{3}{*}{$\mathrm{EV} \rightarrow \mathrm{SAT} \rightarrow \mathrm{LOY}$} & 1.1143 & 0.0596 & $18.6941 \mathrm{a}$ & 0.9969 & 1.2317 & 0.000 \\
\hline & 0.6027 & 0.0732 & $8.2353 b$ & 0.4586 & 0.7467 & 0.000 \\
\hline & 0.5117 & 0.0891 & --- & 0.3470 & 0.6930 & --- \\
\hline \multirow[t]{3}{*}{$\mathrm{SV} \rightarrow \mathrm{SAT} \rightarrow \mathrm{LOY}$} & 0.9756 & 0.0505 & $19.3327 \mathrm{a}$ & 0.8763 & 1.0750 & 0.000 \\
\hline & 0.5396 & 0.0651 & $8.2845 b$ & 0.4113 & 0.6678 & 0.000 \\
\hline & 0.4361 & 0.0736 & --- & 0.3016 & 0.5869 & --- \\
\hline \multirow[t]{3}{*}{$\mathrm{FV} \rightarrow \mathrm{SAT} \rightarrow \mathrm{LOY}$} & 0.8508 & 0.0807 & $10.5439 a$ & 0.6919 & 1.0096 & 0.000 \\
\hline & 0.1087 & 0.0796 & $1.3663 b$ & -0.0480 & 0.2653 & 0.1730 \\
\hline & 0.7421 & 0.0700 & --- & 0.6100 & 0.8832 & --- \\
\hline \multirow[t]{3}{*}{$\mathrm{BV} \rightarrow \mathrm{SAT} \rightarrow \mathrm{LOY}$} & 0.8534 & 0.0716 & $11.9227 \mathrm{a}$ & 0.7124 & 0.9943 & 0.000 \\
\hline & 0.4265 & 0.0574 & $7.4238 b$ & 0.3134 & 0.5396 & 0.000 \\
\hline & 0.4269 & 0.0762 & --- & 0.2909 & 0.5876 & --- \\
\hline
\end{tabular}

注意: SV为社会价值, $\mathrm{EV}$ 为情感价值, $\mathrm{FV}$ 为功能价值, $\mathrm{BV}$ 为品牌价值, SAT为旅游者满意, LOY 为游客忠诚。 $a$ 为总效应, $b$ 为直接效应。 
第二，厘清了古村落游客感知价值、满意和忠诚之间的关系，证实了价值满意因果链， 游客感知价值是游客满意的重要前因。社会价值和情感价值对游客满意和游客忠诚均有显著 的正向影响, 而品牌价值仅对游客忠诚有显著正向影响, 功能价值仅对游客满意有显著正向 影响, 游客满意也显著影响游客忠诚。本研究与汪侠 ${ }^{[21]}$ 、郭安禧 ${ }^{[2]}$ 等人的研究发现一致, 游 客在旅游目的地获得的感知价值越高, 今后重游的可能性会越大。与赵否等研究结果也是比 较接近的, 即生态旅游景区游客感知价值可强化游客忠诚 ${ }^{[48]}$ 。游客满意与忠诚的关系与多数 学者研究结果也是一致的, 满意的游客很可能有重游意向和推荐意愿。根据期望不一致理论, 游客在古村落购买和感受景区提供的产品和服务之后, 体验到的旅游经历与预期旅游体验质 量之间差距越小，或者高于预期，游客则越有可能做出满意的评价，最终会使游客向身边朋 友大力推荐或者再次重游。

第三, 游客满意在社会价值、情感价值和品牌价值与游客忠诚的影响起着部分中介的作 用, 游客满意在功能价值与忠诚的之间具有完全中介的作用。该结论与黄福才和黄颖华、汪 侠和梅虎、赵否等的研究结果是比较相似的。黄福才和黄颖华研究发现, 感知价值是驱动目 的地忠诚最为关键的因素, 它在直接驱动忠诚的同时,还通过满意间接影响目的地忠诚,总驱动 效应最大 ${ }^{[20]}$ 。汪侠和梅虎研究表明, 顾客感知质量、顾客感知价值不仅对顾客忠诚有着直接 的正向影响,还通过顾客满意这一变量间接地对顾客忠诚起着影响作用 ${ }^{[21]}$ 。赵磊等则提出, 生 态旅游景区游客感知价值可以通过提高游客满意来积极影响游客忠诚 ${ }^{[48]}$ 。但是他们将游客感 知价值作为一个整体进行分析，没有反映游客感知价值各个维度的作用。

\section{2 管理启示}

根据以上研究结论，本文提出以下对策建议，以促使我国古村落旅游健康发展。

第一，古村落旅游管理者应该努力提升游客的感知价值。重视社会价值、情感价值对满 意和忠诚直接影响, 关注功能价值对满意的作用, 强化品牌价值对忠诚的直接效应。对于古 村落里满足游客功能需求的产品或服务, 游客关注的更多是功能和价格, 这就要求古村落管 理者能够提供满足游客需求的物美价廉的产品或服务, 既要保质, 又要保量。而对于游客情 感价值的需求, 游客消费更多的是出于情感的需要, 古村落管理者则需要精心设计创造良好 的环境和氛围, 使游客得到与众不同、身心愉悦的体验, 并对古村落产生心理依赖和情绪感 染, 进而提高旅游者满意程度以及忠诚。

第二，古村落管理者要重视古村落的品牌价值。在当今信息化网络化的时代中，品牌代 表的是消费者对产品的认知程度, 而对古村落来说, 也是其最重要的隐形资产。古村落管理 者应该充分地重视其品牌建设, 设计与其他古村落有区别的而能代表其文化底蕴的名称、文 字、标识等。其次, 古村落管理者应该通过各种渠道, 比如报纸、电视、网络等进行积极营 销活动来传递其品牌文化, 方便游客能够通过多种途径了解到古村落旅游的相关信息。最后 将古村落的品牌融进旅游者心中, 使更多的人认识并了解这个地方, 促使其产生旅游动机, 并由古村落潜在旅游者转变成现实旅游者。

第三，古村落管理要注重提高游客满意水平，提升游客忠诚。首先，古村落管理者应该 保护好古村落内部的古建筑以及古村落整体的生态环境, 充分挖掘古村落的独特风俗, 着力 为游客提供独特的旅游体验; 其次尽力保证古村落外部交通良好的通达性, 确保内部标志标 牌醒目, 指示明确; 再次, 重视古村落配套的硬件设施的建设, 比如古村落停车场、洗手间、 休憩设施等要满足游客的需求, 充分考虑旅游高峰期这类需求旺盛时的管理措施和调控措施, 洗手间要结合古村落游客男女比例和女性生理特点及第三类人群的需要来建造; 最后, 建议 古村落游客中心为游客提供超值旅游服务, 尽力满足游客所需要的相关信息和服务, 并为其 提供专业的解说。 


\section{3 研究局限及展望}

本文存在主要的局限为问卷数量不是很多, 古村落只选取了自力村一个古村落, 研究结 论对其他行业的适用性有待进一步验证。除了感知价值、旅游者满意对忠诚有影响以外，是 否还存在其他因素影响，如目的地形象、旅游质量等，还值得未来进一步研究探讨。

\section{致谢}

本文为广东省自然科学基金项目（2016A030313655）和广东财经大学研究生创新项目重 点项目的阶段性成果之一。

\section{References}

[1] V. A. Zeithaml, Consumer Perceptions of Price, Quality, and Value: A Means-end Model and Synthesis of Evidence, Journal of Marketing, vol.52, pp.2-22, 1988.

[2] J. N. Sheth, I. Bruce and Newman, Why we buy what we buy: A theory of consumption values, Journal of Business Research, vol.22, pp.159-170, 1991.

[3] R. B. Woodruff, Customer value: The next source for competitive advantage, Journal of the Academy of Marketing Science, vol.25, pp.139-153,1997.

[4] J. C. Sweeney and G. Soutar, Consumer perceived value: the development of multiple item scale, Journal of Retailing, vol.77, pp.203-220, 2001.

[5] J. C. Sweeney, G. N. Soutar and L. W. Johnson, The role of perceived risk in the quality-value relationship: a study in a retail environment, Journal of Retailing, vol.75, pp.77-105, 1999.

[6] J. F. Petrick, Experience use history as a segmentation tool to examine golf travelers' satisfaction, perceived value and repurchase intentions, Journal of Vacation Marketing, vol.8, pp.332-342, 2002.

[7] Y. H. Huang and F. C. Huang, Tourists' perceived value model and its measurement: An empirical study, Tourism Tribune, vol.8, pp.42-47, 2007.

[8] L. Wang, H. M. Zhang, L. Lu, et al., Tourist perceived value of wetland park: Evidence from Xixi and Qinhu Lake, Tourism Tribune, vol. 29, pp.87-96, 2014.

[9] N. K. Prebensen, H. Kim and M. Uysal, Cocreation as moderator between the experience value and satisfaction relationship, Journal of Travel Research, vol.55, pp.934-945,2015.

[10] A. Parasuraman and D. Grewal, The impact of technology on the quality-value-loyalty chain: A research agenda, Journal of the Academy of Marketing Science, vol.28, pp.156-174, 2000.

[11]C. H. Bai, Literature review of customer value and its implications, Nankai Business Review, vol.2, pp.51-55,2001.

[12]J. J. Cronin, M. K. Brady and G. T. M. Hult, Assessing the effects of quality, value and customer satisfaction on consumer behavioral intentions in service environments, Journal of Retailing, vol.76, pp.193-218,2000.

[13]R. Bolton and J. Drew, A multistage model of customer assessments of service quality and value, Journal of Consumer Research, vol.17, pp.375-384, 1991.

[14]G. S. Day, A two dimensional concept of brand loyalty, Journal of Advertising Research, vol.9, pp.29-35,1969. 
[15]A. S. Dick and K. Basu, Customer loyalty: Toward an integrated conceptual framework, Journal of the Academy of Marketing Science, vol.22, pp.99-113,1994.

[16]J. Jacoby and R. W. Chestnut, Brand loyalty: measurement and management, New York: John Wiley \&Sons, 1978.

[17] S. S. Huang, The effects of motivation, past experience, perceived constraint, and attitude on tourist revisit intention, Hong Kong: Hong Kong Polytechnic University, 2007.

[18]P. Murphy, M. P. Pritchard and B. Smith, The destination product and its impact on traveller perceptions, Tourism Management, vol.21, pp.43-52,2000.

[19]L. J. Su and F. C. Huang, Study on the mechanism of service fairness influencing to consumer loyalty, Journal of Shanxi Finance and Economics University, vol.7, pp.94-103,2010.

[20]F. C. Huang and Y. H. Huang, Research on the driving forces of tourist destination loyalty: A case study of mainland visitors' Hong Kong tours. Tourism Science, vol.3, pp.72-78,2007.

[21]X. Wang and H. Mei, Customer loyalty in tourism destination model and empirical research, Tourism Tribune, vol.10, pp.33-38,2006.

[22]A. X. Guo, Y. Z. Guo, H. J. Li, et al., Empirical research on tourists perceived value influence to revisit intention: The role of tourists' satisfaction and risk probability, Tourism Tribune, vol.33, pp.63-73,2018.

[23]B. Ranjbarian and J. K. Pool, The impact of perceived quality and value on tourists' satisfaction and intention to revisit Nowshahr city of Iran, Journal of Quality Assurance in Hospitality \&Tourism, vol.16, pp.103-117,2015.

[24] Y. H. Kim, M. Kim, T. Ruetzler, et al. An examination of festival attendees' behavior using SEM, International Journal of Event and Festival Management, vol.1, pp.86-95,2010.

[25]T. M. Cheng and C. C. Lu, Destination image, novelty, hedonics, perceived value, and revisiting behavioral intention for island tourism, Asia Pacific Journal of Tourism Research, vol.18, pp.1-18,2013.

[26] J. F. Petrick, D. D. Morais and W. C. Norman, An examination of the determinants of entertainment vacationers intentions to revisit, Journal of Travel Research, vol.40, pp.41-48,2001.

[27]L. J. Su and F. C. Huang, Study on the relationship between tourists' satisfaction and loyalty: A comparative analysis of sightseeing tourists and rural tourists, Tourism Tribune, vol.26, pp.39-45,2011.

[28]G. Assaker, V. E. Vinzi and P. O'Connor, Examining the effect of novelty seeking, satisfaction, and destination image on tourists' return pattern: A two factor, non-linear latent growth model, Tourism Management, vol.32, pp.890-901,2011.

[29]CG-Q. Chi and H. Qu, Examining the structural relationships of destination image, tourist satisfaction and destination loyalty: an integrated approach, Tourism Management, vol.29, pp.624-636,2008.

[30]P. Mechinda, S. Seriat and N. Gulid, An examination of tourists' attitudinal and behavioral loyalty: comparison between domestic and international tourists, Journal of Vacation Marketing, vol.15, pp.129-148,2009.

[31] J. L. Han and F. X. Wei, Study on the interactive relationship between customer satisfaction and loyalty, Nankai Business Review, vol.29, pp.8-10,2001. 
[32] S. Baloglu, An empirical investigation of attitude theory for tourist destinations: A comparison of visitors and nonvisitors, Journal of Hospitality \& Tourism Research, vol.22, pp.211-224,1998.

[33]C. F. Chen and D. Tsai, How destination image and evaluative factors affect behavioral intentions? Tourism Management, vol.28, pp.1115-1122,2007.

[34]H. Z. Alsabbahy, Y. Ekinci and M. Riley, An investigation of perceived value dimensions: Implications for hospitality research, Journal of Travel Research, vol.42, pp.226-234,2004.

[35] V. A. Quintal and A. Polczynski, Factors influencing tourists revisit intentions, Asia Pacific Journal of Marketing and Logistics, vol.22, pp.554-578,2010.

[36] W. B. Li, Study on tourist loyalty model of ancient village: Based on the tourist perceived value and its dimensions, Geographical Research, vol.30, pp.37-48,2011.

[37] S. Um, K. Chon and Y. H. Ro, Antecedents of revisit intention, Annals of Tourism Research, vol.33, pp.1141-1158,2006.

[38]J. Hutchinson, F. Lai and Y. Wang, Understanding the relationships of quality, value, equity, satisfaction, and behavioral intentions among golf travelers, Tourism Management, vol.30, pp.298-308,2009.

[39]M. G. Gallarza and I. G. Saura, Value dimensions, perceived value, satisfaction and loyalty: an investigation of university students' travel behavior, Tourism Management, vol.27, pp.437-452,2006.

[40]X. Wang, C. L. Gu and H. Mei, Tourist attraction customer satisfaction index model, Acta Geographica Sinica, vol.5, pp.807-816,2005.

[41]B. Wang, An empirical study of the relationship between destination image, perceived value, satisfaction and destination loyalty, Tourism Science, vol.25, pp.61-71,2011.

[42] S. M. Rasoolimanesh, N. Dahalan and M. Jaafar, Tourists' perceived value and satisfaction in a community-based homestay in the Lenggong Valley World Heritage Site, Journal of Hospitality and Tourism Management, vol.26, pp.72-81,2016.

[43]X. H. Cheng, Research on the competition advantages of forest sceneries based on the customer perceived value, Hangzhou: Zhejiang University,2006.

[44]J. F. Petrick, Development of a multi-dimensional scale for measuring the perceived value of a service, Journal of Leisure Research, vol.2, pp.119-134,2002.

[45]C. F. Chen and F. S. Chen, Experience quality, perceived value, satisfaction and behavioral intentions for heritage tourists, Tourism Management, vol.31, pp.29-35,2010.

[46]K. J. Preacher and A. F. Hayes, SPSS and SAS procedures for estimating indirect effects in simple mediation model, Behavior Research Methods, Instruments, and Computers, vol.36, pp.717-731,2004.

[47]A. F. Hayes, An introduction to mediation, moderation and conditional process analysis: A regression-based approach, New York: Guilford Press, 2013.

[48]L. Zhao, W. Z. Wu, J. Li, et al., The formation mechanism of tourist loyalty in ecotourism scenic spots from the perspective of tourism perceived value: Evidence from Xixi National Wetland Park, Acta Ecologica Sinica, vol.38, pp.7135-7147,2018. 